\title{
Cooperativismo de plataforma: Análisis de las cualidades democráticas del cooperativismo como alternativa económica en entornos digitales
}

\section{Mayo Fuster Morell, Ricard Espelt, Melissa Renau Cano}

RESUMEN: El fenómeno de la economía de plataforma se articula desde modelos con perspectivas distintas que pueden incluir tanto empresas extractivistas como la evolución de nuevos modelos de colaboración a través de una plataforma digital. Mayoritariamente, la literatura de este ámbito se ha centrado en estudiar casos de plataformas extractivistas dejando en segundo plano las alternativas. Este artículo analiza el origen, las fortalezas y los principales retos que caracterizan las plataformas vinculadas al cooperativismo de plataforma y/o a los comunes digitales. Para ello, se ha llevado a cabo un análisis empírico de veintidós plataformas que operan en cuatro sectores claves en la emergencia de la economía de plataforma: el servicio de taxi, el alojamiento de corta estancia, la distribución de alimentos y los servicios domésticos y de cuidado a domicilio. Los resultados de la investigación señalan que el cooperativismo de plataforma se caracteriza por favorecer relaciones horizontales, por desarrollar una infraestructura comunitaria que respeta la privacidad y facilita la participación de los usuarios en la gobernanza de los datos y, finalmente, por atender algunas de las externalidades negativas causadas por la plataforma. Al mismo tiempo, se muestran varios retos como la apertura de datos, la escala de impacto, la capacidad de réplica del proyecto, la sostenibilidad económica, la inclusión e igualdad de género y la cooperación entre agentes.

PALABRAS CLAVE: Economía de plataforma, cooperativas de plataforma, cualidades democráticas, modelos organizacionales.

CLAVES ECONLIT: J54, L31, L32, Z10.

Cómo citar este artículo/How to cite this article: FUSTER, M., ESPELT, R. \& RENAU, M. (2021): "Cooperativismo de plataforma: Análisis de las cualidades democráticas del cooperativismo como alternativa económica en entornos digitales", CIRIEC-España, Revista de Economía Pública, Social y Cooperativa, 102, 5-31. DOI: 10.7203/CIRIEC-E.102.18429.

Correspondencia: Mayo Fuster Morell, Berkman Klein Center for Internet and Society, Harvard University, ORCID: 0000-0002-2708-3016; Ricard Espelt, Internet Interdisciplinary Institute, Universitat Oberta de Catalunya, ORCID: 0000-0002-3724-2084; Melissa Renau Cano, Internet Interdisciplinary Institute, Universitat Oberta de Catalunya, mrenauc@uoc.edu, ORCID: 0000-0002-7541-5417. 


\section{Expanded abstract}

\section{Platform cooperativism: Analysis of the democratic qualities of cooperativism as an economic alternative in digital environments}

The platform economy phenomenon consists of models with different perspectives that can include extractivist companies to the evolution of new collaboration models through a digital platform. Despite the relevance of the categorisation and differentiation of organisational models, in the platform economy, there is a lack of empirical studies that describe what differentiates them and that show whether the so-called "alternatives to platform capitalism" are built through more participatory and democratic organisations.

In this context, this article aims to contribute to the lack of empirical research of alternatives to platform capitalism from a critical perspective. Specifically, this study aims to empirically study how alternatives to platform capitalism are configured in practice and which are their main challenges.

To do this, an empirical analysis was conducted on twenty-two platforms that operate in four key sectors in the emergence of the platform economy: taxi services, short-stay accommodation, food distribution, and domestic and home care services.

The analysis will determine to what extent platform cooperatives comply with the cooperative and pro-democratic principles that are defended in the theoretical literature on platform cooperativism. It will also reveal to what extent platform economy organisations, which are postulated as an alternative to platform capitalism, represent an effective alternative to it.

The analysis is based on the analytical framework for the study of the democratic qualities of digital platforms previously developed by Fuster Morell and Espelt (2019) in which the platforms are interdisciplinarily evaluated in terms of their pro-democratic characteristics through 147 indicators, which are collected in four different subsections: (i) governance, (ii) economic model, (iii) technology and data policies, and (iv) social responsibility and impact.

The data collection has been carried out through virtual ethnography of the web content of the selected platforms and a set of structured interviews. The codebook collects data of both an objective and a more subjective nature. For example, in the economic dimension, questions are asked about the economic sustainability of the project, the use of ethical banking services or whether it is considered that the workers are developing hard skills. The collection of subjective and objective data allows us to understand what the strengths and weaknesses of the alternatives to platform capitalism are. In other words, thanks to their combination, we can differentiate the current and measurable results of their activity, from the views that the interviewees themselves have regarding the organisations to which they belong, and from their short, medium, and long-term objectives. For example, it allows us to differentiate whether 
or not a platform succeeded in creating the type of organisation defined in the mission, the number of jobs projected, the affinity with the principles of cooperativism and respect for labour rights.

The results of the research indicate that platform cooperativism is characterised by favouring horizontal relationships, by developing a community infrastructure that respects privacy and facilitates the participation of users in data governance and, finally, by addressing some of the negative externalities caused by the platform. At the same time, several challenges are shown, such as the opening of data, the scale of impact, the replicability of the project, economic sustainability, inclusion and gender equality, and cooperation between agents.

Despite the limitation of the number of cases, it is noteworthy that this research contributes to the limited existence of empirical studies, mostly focused on case studies such as Fairbnb or SMart. In this sense, the article outlines possible lines of work that could be tested in future studies. For example, the results of the analysis reveal a tension between the democratic configuration (especially around governance and the economic model) and the sustainability of platform cooperatives. The results also point out the main deficiencies of the alternatives to platform capitalism, revealing both possible future research topics and areas of action by public policies.

The article concludes that there are alternatives to platform capitalism, although they face considerable challenges. For example, in terms of scale, most projects are at an early stage in their evolution. It is relevant to mention that many of the platforms that achieve greater geographic expansion are part of federations of entities. Furthermore, from an environmental perspective, the biggest challenge for platform cooperativism is being able to carry out the holistic vision that the impact of digital technology entails. Even though a good number of projects promote products and services to improve energy efficiency and responsible consumption, only a small portion of platforms are hosted on servers powered by green energy.

Finally, platform cooperatives promote a democratic governance and economic model, based on technology policies around FLOSS. However, platform cooperative projects have two main challenges. On the one hand, promoting an open use of data that enriches public knowledge in the field of platform cooperativism. On the other, the scale of impact, the project's capacity for replication, economic sustainability, gender inclusion and equality, and cooperation between agents.

Keywords: Platform economy, platform cooperatives, democratic qualities, organisational models. 


\section{Introducción}

Airbnb y Fairbnb son dos plataformas digitales que ponen en contacto oferta y demanda de alquileres vacacionales u ocasionales. A pesar de compartir funcionalidades, ambas tienen objetivos y modelos de plataforma distintos. Airbnb es una multinacional creada en 2008 que cuenta con aproximadamente 150 millones de usuarios en más de 65.000 ciudades de todo el mundo, mientras que Fairbnb es una cooperativa que, a través de su plataforma -activa desde el año 2019- ha generado comunidades de usuarios en seis ciudades europeas (Venecia, Barcelona, Bolonia, Valencia, Amsterdam y Génova). Airbnb tiene como objetivo principal el beneficio económico que proporciona la interacción entre las personas que la utilizan y Fairbnb se centra en promover lazos sociales entre sus usuarios (Petruzzi et al., 2019). Es decir, mientras que la finalidad principal de Airbnb es el beneficio económico, Fairbnb prioriza el beneficio social. Esta dualidad en la orientación sobre la cual algunas plataformas provisionan sus productos y servicios se produce en casi todas las áreas de actividad socioeconómica. Por ejemplo, Helpling, Uber y Deliveroo tienen como prioridad el beneficio económico en los sectores del trabajo doméstico, movilidad y reparto de productos a domicilio respectivamente; mientras Equal Care Co-op, Cotabo y CoopCycle representan una alternativa con fines sociales en las mismas áreas de actividad.

Todos estos proyectos forman parte de la economía de plataforma, que está basada en la producción y consumo a través de una plataforma digital (Fuster Morell, 2018). La actividad generada por plataformas globales como Uber o Airbnb, basada en la hiper-externalización, se considera una nueva forma de capitalismo extractivista, regulada por una plataforma digital, en lo que se denomina «capitalismo de plataforma» (Srnicek, 2017); mientras que iniciativas como Equal Care Co-op o CoopCycle se proyectan como una alternativa concebida en torno a los principios del cooperativismo, en lo que Scholz (2016) designa como «cooperativismo de plataforma».

La Economía Social y Solidaria (ESS) se define como «un amplio conjunto de organizaciones y empresas que están específicamente orientadas a producir bienes, servicios y conocimiento mientras persiguen objetivos económicos y sociales y fomentan la solidaridad» (Naciones Unidas, 2015). La definición de ESS se alimenta de la conceptualización de las economías sociales y solidarias y se ha extendido en diferentes sectores como la movilidad, la educación, la alimentación o los servicios financieros para atender las necesidades de las comunidades y su desarrollo (Barea, 1990; Calle Collado y Casadevante, 2015; Schwab do Nascimento et al., 2020). Las organizaciones de ESS promueven actividades económicas a través de relaciones de solidaridad, reciprocidad, autoorganización, articulación, autogestión, cooperación e inclusión; evitando así los problemas económicos, sociales y políticos generados por la economía capitalista neoliberal (Singer, 1998; Laville, 2004; Lechat, 2007). En contrapartida al capitalismo, la ESS permite la construcción de un mercado social, que es democrático, ecológico y de intercooperación por naturaleza (Garcia, 2002). Es decir, la ESS, en su conjunto, se erige como una alternativa al capitalismo. 
La cooperativa como instrumento organizativo de la ESS permite la configuración de empresas democráticas en las que, a diferencia de las empresas capitalistas, las decisiones están en poder de los miembros de la entidad, con igualdad de derecho a voto y evitando la acumulación de capital (Monzón, 2003). Los siete principios sobre los cuales se establecen las cooperativas son: (i) la membresía voluntaria y abierta, (ii) el control democrático, (iii) la participación económica, (iv) la autonomía e independencia, (v) la educación, (vi) la formación e información, y (vii) la cooperación entre cooperativas y la preocupación por la comunidad. Estos principios, que fueron formulados en 1844 por la cooperativa de Rochdale, han sido adoptados a lo largo de la historia por distintos tipos de cooperativas y constituyen el eje vertebral de la Alianza Cooperativa Internacional (Monzón et al., 1989).

Actualmente, en el marco de la sociedad red (Castells, 1996), la gestión de las organizaciones se está reconfigurando a partir del impacto causado por la emergencia y adopción de las Tecnologías de la Información y la Comunicación (TIC). Desde una perspectiva económica, la economía de plataforma ha creado altas expectativas de sostenibilidad debido a su potencial para contribuir al desarrollo sostenible de la sociedad (Botsman y Rogers, 2011; Heinrichs, 2013; Cohen y Kietzmann, 2014). Sin embargo, la economía de plataforma también ha generado una gran controversia debido a los diferentes tipos de modelos surgidos (Codagnone et al., 2016) y sus respectivas correlaciones con la creación de externalidades negativas. Como ya hemos anticipado, aunque a veces no hay discrepancia cuando se menciona la economía de plataforma, es importante destacar que existen diferentes modelos. Ellos van desde los altamente extractivos, asociados a nuevas formas de capitalismo de plataforma, hasta los más colaborativos, como las cooperativas de plataforma (Srnicek, 2017). Por este motivo, la caracterización entre modelos es un punto clave, ya que el diseño de una plataforma digital está correlacionado con su modelo de sostenibilidad y su impacto social, económico y ambiental (Fuster Morell y Espelt, 2019).

No obstante, a pesar de la relevancia de la categorización y la diferenciación de modelos organizacionales, en la economía de plataforma faltan estudios empíricos que describan qué los diferencia y que muestren si las autodenominadas "alternativas al capitalismo de plataforma" se construyen a través de organizaciones más participativas y democráticas. El análisis empírico de la economía de plataforma (exceptuando casos paradigmáticos como las comunidades FLOSS y Wikipedia) y el debate público se han centrado principalmente en el estudio de plataformas globales como Deliveroo, Uber y Airbnb (Vandaele et al., 2019; Cheng y Foley, 2018). De hecho, si retomamos los casos de Airbnb y Fairbnb para ilustrar este aspecto, observamos que en Google Scholar existen 61.900 referencias sobre la primera plataforma, pero solo 200 sobre la segunda. Así mismo, al revisar los resultados en Web of Science (WoS) las cifras son todavía más ilustrativas: para Airbnb una búsqueda temática proporciona 825 resultados, mientras que para Fairbnb solo se dispone de dos. Otra carencia de los estudios de cooperativismo de plataforma es que estos se han centrado mayoritariamente en aportar marcos de análisis o de principios organizativos (Scholz, 2016; Bauwens, 2014), pero pocos son los estudios que cuentan con análisis empíricos de casos (Como et al., 2016; Espelt, 2018). 
En este contexto, el presente artículo tiene como objetivo contribuir a esta carencia de estudios empíricos de alternativas al capitalismo de plataforma desde una perspectiva crítica. Para ello, se realizará un análisis empírico de veintidós plataformas que operan en los cuatro principales sectores económicos, que permitirá determinar dos aspectos. Por una parte, hasta qué punto las cooperativas de plataforma cumplen con los principios cooperativistas y pro-democráticos que se defienden en la literatura teórica sobre el cooperativismo de plataforma. Por otra, hasta qué punto las organizaciones de economía de plataforma, que se postulan como una alternativa al capitalismo de plataforma, suponen una alternativa efectiva para el mismo.

Dicho lo anterior, la siguiente sección se centra en explicar y analizar los principales estudios que se han realizado sobre cooperativismo de plataforma y sus limitaciones. En consecuencia, se presentará el marco de análisis a través del cual se estudiarán veintidós casos de plataformas que se postulan como alternativa al capitalismo de plataforma, la metodología utilizada y el criterio de selección de la muestra, los resultados y, finalmente, nuestras conclusiones principales y propuestas de futuras líneas de investigación.

\section{Cooperativismo de plataforma: definición y antecedentes}

Los estudios de la Economía Social y Solidaria (ESS) señalan la expansión del cooperativismo y algunas de sus cualidades respecto a su capacidad para promover la sostenibilidad social y económica. Según datos oficiales de 156 países, 279,4 millones de estas personas trabajan en cooperativas (como empleados o miembros trabajadores) lo que representa un 9,46\% de las personas empleadas en el mundo (Eum, 2017). Según datos de 2013, las 300 cooperativas y mutualidades más grandes del mundo informaron de una facturación total de 2.360 mil millones de dólares estadounidenses (World Co-operative Monitor, 2015). Por otro lado, los estudios de la ESS señalan una mayor capacidad de recuperación y mejores condiciones de trabajo de los modelos cooperativos en comparación con las empresas tradicionales (Roelants et al., 2012; Birchall y Ketilson, 2009), con fundamento en la evidencia econométrica que existe sobre el comportamiento de las cooperativas de trabajadores y las empresas capitalistas (Burdín y Dean, 2009).

También hay un contraste respecto a la estabilidad laboral entre las cooperativas y el resto de modelos organizacionales (Delbono y Reggiani, 2013). En particular, se ha observado que las actividades intensivas en mano de obra tienden a ser los sectores donde las cooperativas funcionan mejor (Cheney et al., 2014), y las empresas que son propiedad de los miembros tienden a proporcionar puestos de trabajo no cubiertos por el resto de organizaciones del mercado laboral (Birchall, 2012; Bonin et al., 1993; Díaz-Foncea y Marcuello, 2014). Al mismo tiempo, la comparación entre el número de empresas capitalistas y de cooperativas creadas anualmente, evidencia que la creación de cooperativas de trabajadores está relacionada con el porcentaje de desempleo (Díaz-Foncea y Marcuello, 2015). Esta evidencia sugiere que las cooperativas de 
plataforma podrían ser un modelo para la expansión de la economía de plataforma orientada al intercambio, particularmente a través de plataformas laborales, lo que puede generar una mejor sostenibilidad e igualdad social, especialmente en ciudades con alto desempleo.

El término «cooperativismo de plataforma» fue acuñado por el profesor Trebor Scholz en un artículo de 2014 titulado «Platform Cooperativism vs. the Sharing Economy» (Scholz, 2014), en el cual criticaba las plataformas de economía de plataforma extractivista y pedía la creación de alternativas cooperativas controladas democráticamente para que los trabajadores pudiesen intercambiar su trabajo sin la manipulación de un intermediario. Según Scholz, las características principales que definen una plataforma cooperativa son diez: (i) la propiedad colectiva, (ii) el pago digno y la seguridad de ingresos de sus trabajadores, (iii) la transparencia y la portabilidad de los datos creados, (iv) la apreciación y el reconocimiento del valor generado en la actividad de la plataforma, (v) la toma colectiva de decisiones, (vi) un marco legal protector, (vii) la protección transferible de los trabajadores y la cobertura de beneficios sociales, (viii) la protección contra conductas arbitrarias en el sistema de calificación, (ix) el rechazo de la supervisión excesiva en el lugar de trabajo y, finalmente, (x) el derecho de los trabajadores a desconectarse (Scholz, 2016). Posteriormente, el concepto se ha ido popularizando a través de publicaciones editoriales (Scholz, 2016; Scholz y Schneider, 2017), de la organización de una conferencia anual que, a partir de 2015, adoptó el término "platform cooperativism», y de la fundación de un consorcio de actores afines en 2016. El cooperativismo de plataforma promueve las plataformas digitales basadas en los siete principios cooperativos que aseguran su gestión democrática (Scholz, 2016). Por este motivo, dentro de las cooperativas de plataforma se incluyen organizaciones que tienen como prioridad asegurar las condiciones laborales de sus trabajadores y la promoción de un impacto social positivo.

La formulación del concepto del cooperativismo de plataforma tiene varios antecedentes y vasos comunicantes. De hecho, el cooperativismo de plataforma es el término más popular pero no el primero en señalar una coincidencia entre cooperativismo y bienes comunes digitales. Previas, y similares investigaciones sobre nuevas formas de cooperativismo como el cooperativismo abierto (Bauwens y Kostakis, 2014), y también estudios sobre cómo el entorno digital abre nuevas posibilidades para la tradición cooperativa (Murray, 2010; Peuter y Dyer-Witheford, 2010), son de gran importancia en este campo relativamente nuevo. En 2012, la federación cooperativa italiana Legacoop promulgó un manifiesto sobre la "Cooperativa de los Comunes" (Legacoop, 2012) que pedía traer las lecciones del movimiento cooperativo para controlar los datos en línea. El mismo año, Fuster Morell publicó un artículo titulado "Horizontes del procomún digital" (Fuster Morell, 2012) en el que señala la evolución de la producción entre pares basada en bienes comunes que se fusiona con las cooperativas y la economía social. El artículo reflexiona sobre un evento llamado Building Digital Commons, que tuvo lugar en octubre de 2011. Su objetivo fue conectar aún más la tradición cooperativa y la producción colaborativa. Este nuevo tipo de relación entre los principios del cooperativismo y las Tecnologías Información y Comunicación (TIC), en el caso de adoptar el software libre de código abierto (FLOSS) y las licencias abiertas (Fuster Morell et al., 2016), lleva al cooperativismo de plataforma a un modelo cooperativo abierto (Bauwens, 2014; Bauwens y Kostakis, 2015; Pa- 
zaitis et al., 2017). De igual manera, estudios sobre cómo el entorno digital abre nuevas posibilidades para la tradición cooperativa apuntan a un lugar de encuentro entre el cooperativismo y la adopción de las TIC (Peuter y Dyer-Witheford, 2010; Murray, 2010).

A pesar del desarrollo conceptual y la aparición de plataformas en las principales áreas socioeconómicas, el cooperativismo de plataforma, probablemente debido a su novedad, cuenta con limitados estudios empíricos que analicen su potencial transformador de acuerdo con los siete principios cooperativistas. Como et al. (2015) desarrollaron un análisis exploratorio, basado en entrevistas con cooperativas, primero en Italia y luego en nueve países europeos, sobre las actitudes de las cooperativas hacia la economía de plataforma y un mapeo de encuestas en línea de cooperativas que están implementando innovaciones en el campo de la economía de plataforma (Como et al., 2016). Las entrevistas encontraron una actitud positiva predominante por parte de los entrevistados de las organizaciones cooperativas hacia la idea de desarrollar innovaciones a través de la economía de plataforma, aunque también hubo opiniones críticas en algunos países como Italia y los Países Bajos. Las federaciones de cooperativas en el Reino Unido, Austria y Bélgica tienen programas para estimular las plataformas cooperativas. Más adelante, Martinelli et al. (2019) publicaron una investigación en la que presentaban diferentes casos de cooperativas de plataforma, entreviendo su potencial para crear y fomentar puestos de trabajo de calidad haciendo uso de plataformas digitales pero sin depender exclusivamente de su adopción. No obstante, el artículo no analiza cómo cada una de las organizaciones presentadas logra, o no, mejorar las condiciones de trabajo y el resto de cualidades democráticas; sino que simplemente entrevé su potencialidad (ibid). El presente artículo parte de un análisis empírico previo de cien plataformas (Fuster Morell y Espelt, 2019) y de algunos estudios de caso sobre cooperativas de plataforma focalizados en proyectos muy paradigmáticos como Fairbnb (Foramitti et al., 2020; Petruzzi et al., 2019) o SMart (Charles et al., 2020).

Estas primeras investigaciones enfatizan el rol de la gobernanza en la articulación de una plataforma digital. El desarrollo de la ideación y la evolución de Fairbnb se correlacionan con el debate sobre cómo se gobierna la plataforma (Foramitti et al., 2020). En esta misma línea, sobre SMart se evalúa el impacto que tiene su organización en las condiciones de trabajo de sus socios y el papel que tiene la gobernanza de la plataforma en el fomento de mejores condiciones laborales (Charles et al., 2020). Finalmente, Fuster Morell y Espelt (2019), con base en el estudio empírico de cien plataformas de economía de plataforma, destacan la correlación entre el modelo de gobernanza de la plataforma y su modelo de sostenibilidad. Al mismo tiempo, sobre el mismo trabajo de campo, se observa una correlación entre el diseño de una gestión democrática y la promoción de unas políticas tecnológicas y de datos abiertas (Fuster Morell y Espelt, 2018).

En el presente artículo tomamos como referencia las investigaciones precedentes y contribuimos al estudio de las cualidades democráticas de la economía de plataforma, que describimos en la siguiente sección, para analizar empíricamente veintidós plataformas que se postulan como alternativa al capitalismo de plataforma. 


\section{Marco de análisis}

Las cualidades democráticas para el análisis de plataformas digitales formuladas con base en la conceptualización del cooperativismo de plataforma y sus vasos comunicantes, se articulan en torno a las dimensiones de gobernanza, modelo económico, políticas tecnológicas, políticas de datos, responsabilidad social e impacto (Figura 1). Éstas tienen en consideración dos aspectos. Por un lado, las cualidades democráticas que provienen de la literatura del cooperativismo (Ratner, 2009) que, en respuesta al capitalismo, apuntan a la importancia de los vínculos que existen entre la gobernanza y el modelo económico, y entre el impacto y la responsabilidad social. Por otro lado, los estudios de procomún tanto de la escuela de 0strom, que ponen el acento en la gobernanza (Ostrom, 2015), como de la escuela de Cyber Scholar, que ponen el acento en las condiciones de acceso y las políticas de propiedad del conocimiento (Benkler, 2006; Lessig, 1999; Rose, 1986).

\section{Figura 1. Marco analítico para el estudio de las cualidades} democráticas de las plataformas digitales

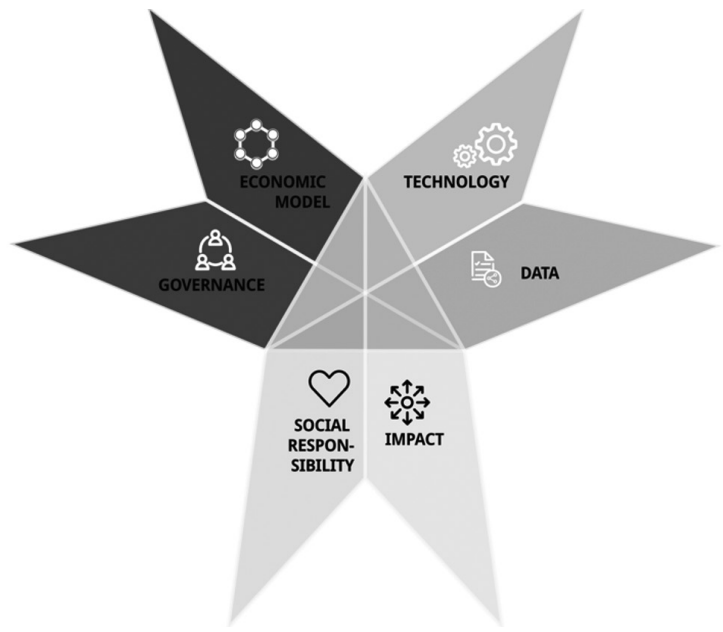

Fuente: Fuster Morell, Espelt, 2019.

Cada una de estas dimensiones es estudiada a través de un libro de códigos formado por 147 indicadores (Dimmons, 2019).

La gobernanza considera el nivel de interacción que la plataforma permite a través de sus funcionalidades y la participación democrática con respecto a la organización que la promueve. 
El modelo económico analiza la orientación económica del proyecto, la sostenibilidad, los modelos de financiación y las relaciones laborales derivadas del uso de la plataforma.

Las políticas tecnológicas verifican la licencia sobre la cual se ha desarrollado la plataforma y su arquitectura que determinan la capacidad de réplica del proyecto.

Las políticas de datos inciden en la licencia que regula los contenidos de la plataforma, su acceso y gobernanza.

La responsabilidad social incluye la equidad de género, la inclusividad, la responsabilidad ambiental y el respeto a la soberanía de la ciudad para regular la actividad de la plataforma.

Finalmente, la evaluación del impacto considera el nivel de desarrollo del proyecto, el nivel de sostenibilidad económica, el nivel de uso y la capacidad de cooperación con otros agentes.

\section{Metodología}

Esta investigación se centra en el análisis de veintidós plataformas digitales que se postulan como alternativa a las plataformas extractivistas, también denominadas como "unicornio" (e.g. Fuster Morell y Espelt, 2019), en cuatro de las áreas en donde más se han hecho visibles los impactos de la hiper-externalización que las plataformas extractivistas provocan: (i) la transformación del sector del taxi (Uber), (ii) el alojamiento de corta estancia (Airbnb), (iii) la mensajería (Deliveroo), y (iv) los servicios domésticos y de cuidado a domicilio (Helpling). Las plataformas que ostentan el liderazgo de estos cuatro sectores (Uber, Airbnb, Deliveroo o Helpling, entre las más destadadas) comparten el hecho de postular que operan como «meras agregadoras» de oferta y demanda, situándose como terceras partes en una relación económica, pero contribuyen a la extrema subcontratación de bienes y servicios, fomentando así la degradación de las condiciones laborales (Srnicek, 2017).

Ante este contexto, son varias las iniciativas que, con distintas formas jurídicas, emergen con la intención de ofrecer una alternativa a este tipo de plataformas. No obstante, como se ha comentado en la introducción de este artículo, son necesarios estudios empíricos que abarquen esta diversidad de «alternativas», sus retos y fortalezas, y analicen hasta qué punto promueven valores más democráticos y/o basados en los principios del cooperativismo. Por este motivo, más allá que su formato jurídico sea o no el de cooperativa, la selección de casos ha tenido en cuenta que las iniciativas no tengan una voluntad extractivista y que traten de minimizar las externalidades negativas que provoca su actividad.

El análisis se basa en el marco analítico para el estudio de las cualidades democráticas de las plataformas digitales (Figura 1) y concretado a través de un libro de códigos (Dimmons, 2019). La recopilación de datos se ha llevado a cabo a través de una etnografía virtual del contenido web de las plataformas seleccionadas, y de una entrevista estructurada a una figura con poder de decisión y conocimiento transversal (fundadores/as y/o miembros/as activos/as de las cooperativas, directores/as de la empresa, etc.). El libro de códigos recopila datos de indicadores tanto de carácter objetivo como de carácter más subjetivo (basado en las perspectivas de las personas entrevistadas). Por ejemplo, en la dimensión económica, se pregunta sobre la 
sostenibilidad económica del proyecto, el uso de servicios de banca ética o si se considera que los trabajadores están desarrollando habilidades duras. La recopilación de datos subjetivos y objetivos, permite comprender cuáles son las fortalezas y debilidades de las alternativas al capitalismo de plataforma. En otras palabras, gracias a su combinación, podemos diferenciar los resultados actuales y medibles de su actividad, de los puntos de vista que tienen los propios entrevistados respecto a las organizaciones a las cuales pertenecen y de sus objetivos a corto, medio y largo plazo. Por ejemplo, nos permite diferenciar si una plataforma consiguió, o no, crear el tipo de organización definida en la misión, la cantidad de puestos de trabajo esperados, la afinidad con los principios del cooperativismo y el respeto de los derechos laborales.

\section{Resultados y discusión}

El análisis se realizó sobre veintidós plataformas de cuatro áreas claves de la economía de plataforma, a saber: (i) el servicio de taxi (Taxi Ecològic, Taxiapp UK, Alpha Taxis y Cotabo Taxi Bologna); (ii) el alojamiento de corta estancia (Fairbnb, BeWelcome, Les oiseaux de passage, Fairbooking, Trustroots y Holidays Exchange Coop); (iii) el reparto de última milla (La Colmena que Dice Sí, CoopCycle, Open Food Network, Mensakas, La Cour Cyclette, Crow Cycle Courier Collective, Olvo y La régie de quartier de Stains); y, finalmente, (iv) las tareas domésticas y de cuidado a domicilio (Helpific, Qida, Parentalis y Equal Care Co-op).

Figura 2. Tipo de conexión con el entorno digital $(n=22)$

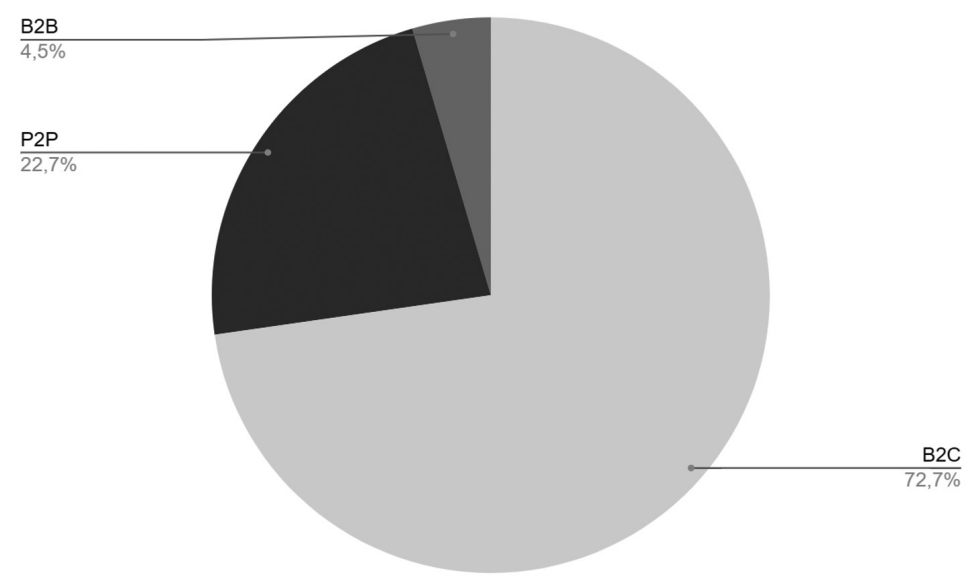

Fuente: Elaboración propia. 
En cuanto al tipo de conexión con el entorno digital que caracteriza a estos veintidós casos, se tiene que, en primer lugar, el 72,7\% articula prácticas comerciales entre empresas y consumidores, y solo el 4,5\% lo hace entre empresas; en segundo lugar, el 22,7\% fomenta relaciones entre pares (Figura 2); y, por último, el 68,2\% no tiene un modelo cuyo principal espacio de interacción sea la plataforma, lo cual es importante destacar ya que ésta es concebida como una herramienta más dentro del modelo organizativo.

\subsection{La gobernanza: el formato jurídico como clave para una gobernanza abierta y transparente}

Del total de veintidós plataformas analizadas, el 54,5\% están promovidas por cooperativas (todas ellas sin ánimo de lucro), el 18,2\% por asociaciones, el 4,5\% por fundaciones y el 22,7\% están impulsadas por una entidad comercial (Figura 3).

\section{Figura 3. Tipología de entidad legal $(n=22)$}

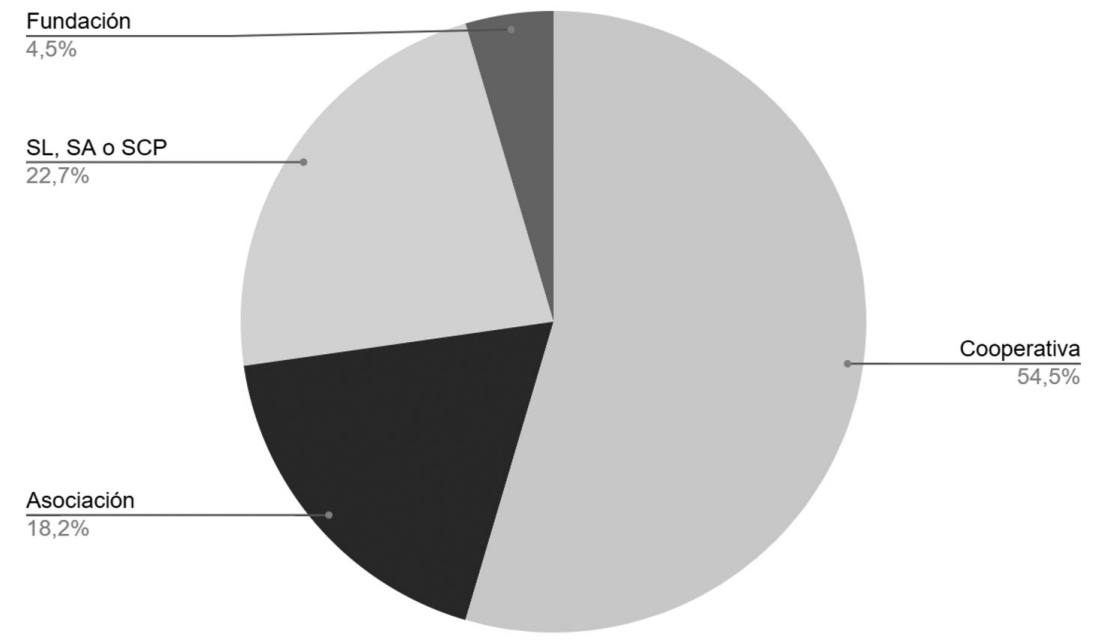

Fuente: Elaboración propia.

Como resultado, el marco legal de la mayoría de la plataformas analizadas $(77,2 \%)$ favorece un modelo de gobernanza participativo. A partir de las trece entrevistas realizadas observamos que siete entidades -con formato de asociación, cooperativa o fundación- facilitan el acceso directo al balance económico a personas socias y tres de ellas lo hacen público directamente, mientras que ninguna de las alternativas bajo una forma jurídica comercial (SL, SA o SCP) lo hacen. Además, estas entidades incentivan que los usuarios participen en la toma de 
decisiones sobre el destino de la distribución de los beneficios económicos. Este, por ejemplo, es el caso de "Crow Cycle Courier Collective", un colectivo de catorce repartidores que utiliza CoopCycle y que, a diferencia del modelo de distribución de tareas de empresas como Glovo o Deliveroo basado en la competencia (Cant, 2020), distribuye el trabajo basándose en la equidad y la solidaridad económica:

Nos consideramos verdaderamente democráticos. Hablamos entre nosotros y si alguien está en una situación financiera difícil y quisiera trabajar más, buscamos la forma de que esta persona pueda trabajar más. No se trata de quién y qué. Es solidaridad y empatía. Así es como encontramos soluciones, incluso si es una solución privada.

(Crow Cycle Courier Collective, noviembre 2019)

En este mismo sentido, y completamente antagónico al modelo extractivista basado en la degradación de los derechos laborales, incluyendo el derecho a la organización colectiva (De Stefano, 2016; Vandaele, 2018; Urzí Brancati et al., 2020), la organización colectiva de los trabajadores se postula como un elemento clave para la gobernabilidad democrática. De hecho, en algunos casos, la creación de la cooperativa fue en sí misma un acto de organización colectiva. Este es el caso de Mensakas, una cooperativa de reparto a domicilio fundada en Barcelona por antiguos repartidores de plataformas como Glovo y Deliveroo.

En concordancia con la literatura sobre las prácticas de la economía social y solidaria, los principios cooperativistas (Monzón et al., 1989) y del cooperativismo de plataforma (Scholz, 2016), los resultados afirman que ciertas formas jurídicas, como las cooperativas, fomentan la toma de decisiones de forma democrática. Éste es un elemento que contrasta con la opacidad y la jerarquía del capitalismo de plataforma (Srnicek, 2017), el cual impide a los usuarios participar en la toma de decisiones (Fuster Morell et al., 2020). En este sentido, once de las trece organizaciones entrevistadas, diez de ellas cooperativas, asociaciones o fundaciones, vinculan a sus miembros en la definición de las normas formales y las políticas de la plataforma; seis de ellas a través de un sistema de toma de decisiones formal (v.gr. asambleas o votaciones) 0 informal (v.gr. espacios de deliberación). No obstante, y en contraste con las organizaciones recogidas bajo formas jurídicas de la Economía Social y Solidaria, ninguna de las empresas bajo formas comerciales involucra ni a los trabajadores ni a los usuarios en la toma de decisiones respecto a la distribución de beneficios económicos.

\subsection{El modelo económico: hacia un modelo económico no especulativo y respetuoso con las condiciones laborales}

En la introducción hemos mencionado que a pesar que Airbnb y Fairbnb ofrecen el mismo servicio, la primera se centra en maximizar el retorno económico al margen de los impactos negativos, mientras que la segunda prioriza una actividad económica basada en criterios éticos 
y de sostenibilidad. Este elemento distintivo se repite en el conjunto de casos analizados. En efecto, el $100 \%$ de las plataformas entrevistadas tienen un modelo de crecimiento que deja de lado la búsqueda del crecimiento exponencial sin escrúpulos característico de empresas como Uber, y da paso a la priorización de la misión del proyecto basada en la promoción de valores democráticos y los principios del cooperativismo. Incluso cinco de las trece plataformas entrevistadas, promueven relaciones no monetarias entre los agentes que las utilizan, por ejemplo, promoviendo el intercambio de vivienda sin intercambio monetario.

Un aspecto clave que nos ayuda a corroborar que su modelo de crecimiento no está basado en el crecimiento exponencial es el tipo de fuentes de financiación que utilizan (Figura 4). Mientras que la fuente de financiación principal para la mayoría de plataformas extractivistas que aspiran a convertirse en "unicornio" son las rondas de financiación (Srnicek, 2017), solo tres de los trece casos estudiados en esta investigación, han recurrido a rondas de financiación u otros medios de financiación privada.

En este sentido, los modelos de financiación más extendidos a lo largo de los trece casos a los que se les realizó una entrevista son la oferta de servicios premium y la comercialización de la marca (12 plataformas), seguidos por la organización de eventos (8), la financiación pública (7) y las cuotas por membresía, y las donaciones no monetarias de la comunidad (6). El resto de modelos de financiación tienen un uso menor, salvo por la publicidad y el uso de monedas alternativas que no tienen presencia: las donaciones de dinero voluntarias (4), el microfinanciamiento y donaciones no monetarias externas (3), la participación en proyectos de investigación tipo H2020 (2) y la explotación de recursos libres (1). Finalmente, es importante indicar que tres de las trece entidades utilizan servicios de banca ética y que cinco de los trece proyectos han participado en programas de emprendimiento con vocación social (Figura 4).

Tal y como se ha comentado en el marco teórico de este artículo, un elemento destacado es la capacidad de las cooperativas para generar puestos de trabajo de calidad (Roelants et al., 2012; Birchall y Ketilson, 2009). En cuatro de las trece plataformas, los trabajadores están contratados por la organización, mientras que en el resto de casos son autónomos o independientes. Por ejemplo, en lo que concierne a las plataformas de reparto, cuatro de un total de seis plataformas entrevistadas o bien combinan ambos tipos de reconocimiento o se basan en un modelo fundamentado en el trabajo por cuenta propia. Esta coexistencia de distintas formas de reconocimiento se debe a que las plataformas abogan por su autonomía de decidir el tipo de modelo que quieren promover y por la falta de recursos económicos. Por otro lado, en lo que atañe a la única organización entrevistada del sector del taxi, ella opera con trabajadores independientes. Luego, respecto a las organizaciones estudiadas dentro del sector de estancias temporales es un tanto especial, puesto que existe cierta relevancia en el intercambio no monetario (tres de las cuatro entrevistadas). Por último, con relación al sector de trabajo doméstico y de cuidados, las dos plataformas entrevistadas contratan a sus trabajadores/as como asalariados. 


\section{Figura 4. Fuentes de financiación $(n=13)$}

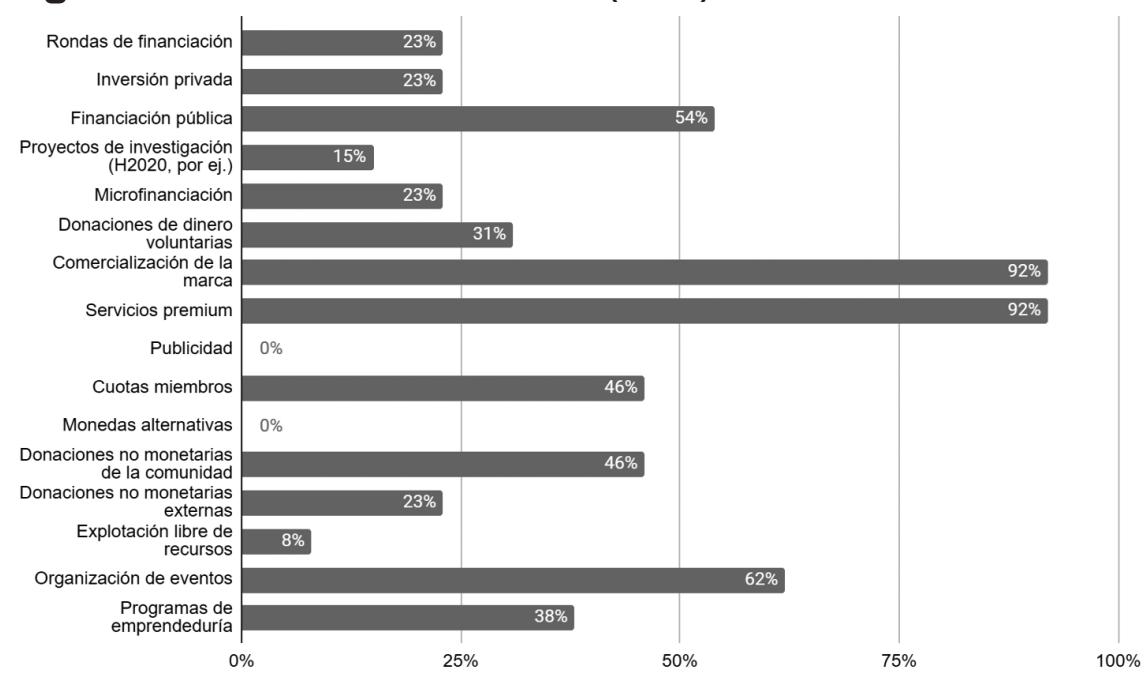

Fuente: Elaboración propia.

Uno de los principales retos que presentan las alternativas analizadas, es que solo tres de las trece plataformas indican que sus trabajadores ganan más del salario mínimo interprofesional (SMI) considerando el costo de la hora de trabajo. Además, solo cinco de las trece plataformas han definido unas políticas de cuidados de forma explícita y/o disponen de un programa de salud para los trabajadores ajeno a las prestaciones públicas.

No obstante, en contraposición a los impactos laborales provocados por la economía de plataforma extractivista (Todolí, 2018; Urzí Brancati et al., 2020) se pueden destacar varios aspectos. En primer lugar, ocho de las trece organizaciones entrevistadas proveen los materiales requeridos para hacer las tareas que se ofertan en la plataforma. En segundo lugar, en relación a la mejora de las competencias laborales y bienestar laboral, se observa un contraste relevante entre los casos analizados y el trato que las plataformas extractivistas dan a este tema. En tercer lugar, doce de las trece plataformas indican que los trabajadores adquieren habilidades duras durante su jornada. Asimismo, diez de las trece indican que las tareas laborales no son repetitivas y en ocho de las trece los trabajadores no deben realizar tareas en tiempos de ejecución ajustados. Finalmente, otro indicador relevante relacionado con el equilibrio entre vida laboral y personal y la tan aclamada "flexibilidad", es que en diez de las trece plataformas, los trabajadores pueden decidir el horario y el número de horas que desean trabajar de forma autónoma e independiente. Esto se complementa con el hecho que ya antes de la COVID-19, seis de las trece podían teletrabajar y solo una plataforma penalizaba por no trabajar durante las horas donde había un pico de la demanda. 


\subsection{Las políticas tecnológicas y políticas de datos}

Respecto al papel de la tecnología en la gestión de relaciones laborales, solo dos de las trece plataformas entrevistadas utilizan o planean utilizar inteligencia artificial, y ninguna realiza regularmente capturas de pantalla de los dispositivos de los empleados. Por otra parte, tres de las trece utilizan técnicas de geolocalización y solo una utiliza técnicas de gamificación. Además, en ocho de los trece casos los trabajadores pueden renunciar a los mecanismos de control sin ser penalizados.

En cuanto a las políticas tecnológicas, seis de las trece plataformas tienen una licencia de programación abierta y siete de las trece tienen una arquitectura tecnológica abierta.

Respecto a las políticas de datos, solo dos de las trece plataformas otorgan una licencia Creative Commons a sus contenidos y solo una de las trece tiene una licencia abierta sobre el acceso a sus datos. Ninguna de las plataformas analizadas permite exportar o copiar los datos generados por la actividad de las mismas. En contrapartida al bajo interés por la apertura de los datos, diez de las trece plataformas fomentan la participación de los usuarios en la gobernanza de los mismos.

\subsection{La responsabilidad social y el impacto: sostenibilidad ambiental, vinculación con la comunidad y políticas a favor de la igualdad}

Inicialmente, la economía de plataforma generaba grandes expectativas respecto a su contribución al desarrollo sostenible de la sociedad (Botsman \& Rogers, 2011; Cohen \& Kietzmann, 2014; Heinrichs, 2013). Sin embargo, más adelante ellas se pusieron en duda debido al impacto de plataformas como Uber y Airbnb (Codagnone et al., 2016). Por ello, resulta interesante analizar el comportamiento de las alternativas analizadas en este aspecto. A nivel de responsabilidad medioambiental, cinco de las trece plataformas promueven el reciclaje y la economía circular, dos de las trece están alojadas en servidores alimentados por energía verde y seis de las trece ofrecen productos o servicios para mejorar la eficiencia energética. En este sentido, la persona entrevistada de Open Food Network comentó tanto la falta de alternativas potentes a las principales infraestructuras capitalistas como la dificultad a su acceso debido al precio. Finalmente, nueve de los trece proyectos entrevistados priorizan proveedores de la economía social y seis de los trece proveen materiales de sensibilización de consumo responsable como, por ejemplo, la repartición de panfletos informativos en alojamientos turísticos.

Una característica que define a empresas "unicornio" como Uber, es su reticencia a colaborar con la administración pública en beneficio de las comunidades locales, alejándose de otros actores del sector a menos que actúen de acuerdo con sus demandas, lo cual provoca daños en los territorios en los que operan (Zwick, 2018). Sin embargo, las alternativas estudiadas muestran ser contrarias a este modus operandi. En efecto, ocho de las trece organizaciones entrevistadas forman parte de una federación de entidades a nivel nacional, internacional y/o 
sectorial; seis de las trece han colaborado con la administración pública, cinco de las trece con organizaciones de investigación, siete de las trece con organizaciones de la sociedad civil, y seis de las trece con organizaciones empresariales. Es decir, se observa un buen nivel de interacción entre actores bajo la perspectiva propuesta por el modelo cuádruple hélice.

Por otro lado, la economía de plataforma extractivista presenta desafíos para la igualdad de género. Diferentes autores sostienen que este modelo reproduce jerarquías y sesgos de género, raza y clase (Edelman \& Luca, 2014; Schor, 2016; Schor et al., 2016), agregando que los algoritmos también reproducen estereotipos de género (Kenny \& Donnelly, 2020). De hecho, esto se confirmó en la gran mayoría de las organizaciones entrevistadas teniendo en cuenta que, en cinco de las trece, se constata que hay equidad de género entre las personas que utilizan la plataforma, en dos de las trece respecto a las personas que trabajan en ella y en ninguna respecto a la propiedad de la plataforma. Estos resultados indican que la equidad de género es un área de trabajo, incluso para los modelos alternativos. No obstante, es importante mencionar que cuatro de las trece iniciativas entrevistadas tienen políticas explícitas para promover la equidad de género, que incluyen medidas como la autorización de la asistencia de menores a las reuniones o un mayor salario para las mujeres. Finalmente, respecto a la inclusión, seis de las trece plataformas analizadas están adaptadas a personas con diversidad funcional, ocho de las trece promueven la inclusión de personas con bajos ingresos, y siete de las trece fomentan la inclusión de personas con nivel educativo básico.

\section{Conclusiones}

La economía de plataforma no solo abarca un rango diverso de actividades económicas (Schor y Fitzmaurice, 2015; Cohen y Muñoz, 2016; Hamari et al., 2016; Martin, 2016; J. Schor, 2016), también engloba una considerable diversidad en cuanto a modelos de negocio y sus respectivos impactos (Fuster Morell y Espelt, 2019; Laukkanen y Tura, 2020). Como ya hemos anticipado, aunque a veces se haga referencia a la economía de plataforma sin reproches, existen modelos más colaborativos y pro-democráticos como las cooperativas de plataforma (Scholz, 2016). En este sentido, el cooperativismo de plataforma surge como un antídoto para la economía de plataforma extractivista con fines de lucro y resultante de la evolución del capitalismo (Srnicek, 2017). El cooperativismo de plataforma favorece la construcción de un movimiento social centrado en prácticas genuinas de intercambio y cooperación en la producción y consumo de bienes y servicios, en base a la democratización de la gobernanza de las plataformas y potenciando la actividad económica colaborativa entre pares (Scholz, 2016; Fuster Morell, 2016). La caracterización y diferenciación de modelos es clave ya que el diseño de una plataforma digital está correlacionado con su modelo de sostenibilidad, su impacto social, económico y ambiental (Fuster Morell y Espelt, 2019).

Esta investigación ha tenido como objetivo estudiar empíricamente cómo se configuran en la práctica las alternativas al capitalismo de plataforma (Srnicek, 2017). El análisis se ha efectuado a partir del marco analítico desarrollado previamente por Fuster Morell y Espelt (2019) 
en el que las plataformas son evaluadas interdisciplinariamente en cuanto a sus características pro-democráticas a través de 147 indicadores, que se recogen en cuatro subapartados diferentes: (i) gobernanza, (ii) modelo económico, (iii) políticas tecnológicas y de datos, y (iv) responsabilidad social e impacto. La definición de estas cualidades democráticas retoman los siete principios del cooperativismo (Monzón et al., 1989) y la voluntad de fomentar un mercado social (Garcia, 2002), adoptando una perspectiva digital en respuesta a las plataformas extractivas (Scholz, 2016), e incorporando el software libre de código abierto (FLOSS) y las licencias abiertas (Fuster Morell et al., 2016) formuladas desde la óptica de un impulso al cooperativismo abierto (Bauwens y Kostakis, 2015). Al mismo tiempo, las cualidades democráticas incorporan una perspectiva feminista y ecológica (Fuster Morell, 2016).

Partiendo del "Marco analítico para el estudio de las cualidades democráticas de las plataformas", y tanto de etnografía virtual como de entrevistas estructuradas, se han analizado veintidós plataformas digitales, de cuatro sectores clave, en los que la economía de plataforma ha provocado un gran impacto socioeconómico: (i) el del servicio de taxi, (ii) el de la oferta de alojamiento de corta estancia, (iii) el de la distribución de alimentos y tareas domésticas, y (iv) el de los cuidados a domicilio. A pesar de la limitación del número de casos, es de destacar que esta investigación contribuye a la reducida existencia de estudios empíricos, en su mayoría focalizados en estudios de caso como Fairbnb (Foramitti et al., 2020) o SMart (Charles et al., 2020). En este sentido, ella no solo permite extraer algunos elementos que pueden ser útiles para vislumbrar la contribución del cooperativismo de plataforma, en el marco de la expansión de alternativas en la economía de plataforma. Además, ella lleva a cabo un análisis crítico de las alternativas de plataforma y deja en evidencia las áreas hacia donde podrían dirigirse, por ejemplo, las futuras políticas públicas.

En resumen, a pesar de las limitaciones en cuanto a la extrapolación y generalización de los resultados, especialmente atendiendo a que el análisis completo de indicadores se ha realizado sobre trece de las veintidós plataformas que conforman la investigación, el artículo traza posibles líneas de trabajo que podrían ser sometidas a prueba en futuros estudios. Por ejemplo, los resultados del análisis vislumbran una tensión entre la configuración democrática (especialmente en torno a la gobernanza y al modelo económico) y la sostenibilidad del cooperativismo de plataforma. Este aspecto es de gran importancia, porque investigaciones previas apuntan al potencial de la economía de plataforma para la creación de valor sostenible atendiendo a los diferentes modelos de plataformas (Laukkanen y Tura, 2020). Los resultados también señalan las principales deficiencias de las alternativas al capitalismo de plataforma, dejando entrever tanto posibles temáticas futuras de investigación, como ámbitos de actuación por parte de políticas públicas enfocadas a los estudios de procomún.

La mayoría de plataformas estudiadas tienen una clara voluntad de articular prácticas comerciales entre empresas y consumidores, ya sea como soporte de una actividad no digital o para favorecer una interacción exclusivamente digital. Es decir, la mayoría de plataformas digitales estudiadas se configuran como un instrumento de intermediación más entre empresas y consumidores, sin que estas sean el eje principal de la interacción. A nivel económico, se observa que todos los proyectos, independientemente de si tienen un modelo de crecimiento 
orgánico o federado, no tienen voluntad extractivista o especulativa. Este aspecto concuerda con las fuentes de financiación, ya que las plataformas estudiadas no recaen en su mayoría en rondas de financiación privada, totalmente contrario a modelos como Deliveroo o Uber donde la dependencia de rondas de financiación es definitiva para su modelo de negocio (Solel, 2019; Zwick, 2018). En su lugar, en ellas tiene un peso importante la financiación pública y el uso de la banca ética. Al mismo tiempo, las organizaciones del cooperativismo de plataforma promueven que los trabajadores tengan mejores condiciones de trabajo, ya que facilitan los materiales y recursos para el desarrollo de la tarea y promueven el desarrollo de habilidades profesionales, entre otros elementos relacionados con las condiciones laborales. No obstante, en cuanto a condiciones económicas se evidencia la dificultad de garantizar puestos de trabajo estables y bien remunerados: solo tres de las trece considera que todos sus trabajadores cobran más o igual al salario mínimo por hora y las formas de empleo por cuenta propia son mayoría (69\%). En contraste con las plataformas extractivistas (De Stefano, 2018; Ivanova et al., 2018; Rosenblat, 2018) y de acuerdo con el análisis realizado, las organizaciones de cooperativismo de plataforma no suelen servirse de algoritmos y/o técnicas de gamificación para controlar y gestionar el comportamiento de los trabajadores.

Respecto a las políticas de datos, se produce un contraste entre la posibilidad que tienen los usuarios de participar en la gobernanza de los datos y la poca posibilidad de acceder a ellos, debido a la ausencia de licencias sobre los datos generados y la incapacidad de exportar o copiar los mismos. En contraste al poco interés por las políticas de datos, la mayoría de las plataformas de cooperativismo analizadas se basan en el código abierto y la arquitectura tecnológica también es abierta. Es decir, mientras observamos una transición del cooperativismo hacia la programación libre de código abierto (FLOSS) (Fuster Morell et al., 2016), la apertura hacia un conocimiento abierto sigue siendo muy limitada. La formación de un cooperativismo de plataforma en torno a un modelo cooperativo abierto (Bauwens, 2014), es aún un reto y un espacio de trabajo relevante.

El análisis muestra algunos resultados, en cuanto a la gobernanza, que gracias a literatura previa gozan de mayor robustez (Fuster Morell y Espelt, 2019; Charles et al., 2020). La gobernanza en las plataformas basadas en el modelo de cooperativismo de plataforma, dirigidas a la descentralización de la toma de decisiones, resulta clave en la creación de alternativas al capitalismo de plataforma. Más allá de que la forma jurídica dominante sea la cooperativa, todas las iniciativas consideran que proporcionan espacios para que las personas que trabajan o interactúan en la plataforma puedan organizarse colectivamente. En algunos casos, incluso el proceso de la creación de la cooperativa supone, en sí mismo, un acto de organización colectiva (v.gr., Mensakas). Un elemento divergente de la investigación es la digitalización, mientras que los resultados indican que gran parte de los proyectos no hacen uso de herramientas digitales para facilitar la participación en toma de decisiones, otras investigaciones en áreas económicas concretas como la energía eléctrica (Riutort Isern, 2016) o la agroecología (Espelt, 2018) indican la importancia que tienen las herramientas digitales en la gobernanza de la plataforma. No obstante, independientemente de las herramientas tecnológicas, las alternativas al capitalismo de plataforma vinculan a la comunidad en la definición de las normas 
formales y las políticas de la plataforma y en la distribución de los beneficios económicos. También es de destacar el nivel de transparencia interna en relación a la actividad económica que la plataforma genera, aunque en este caso las plataformas que operan bajo formas jurídicas comerciales muestran menor grado de transparencia. En este sentido, el cooperativismo de plataforma se caracteriza por (1) favorecer las relaciones horizontales, en contraste con el mando tradicionalmente jerárquico en el gobierno de las plataformas digitales; (2) desarrollar una infraestructura comunitaria que respeta la privacidad y da como resultado la provisión (generalmente) de acceso abierto a recursos comunes que favorecen la accesibilidad, la reproducibilidad y la derivación.

En conclusión, sobre la base de las cualidades democráticas de las plataformas digitales -que considera las dimensiones de (i) gobernanza, (ii) modelo económico, (iii) políticas tecnológicas, (iv) políticas de datos y (v) responsabilidad social de los impactos del proyecto- existen alternativas al capitalismo de plataforma aunque se enfrentan a retos considerables. En cuanto a su escala, la mayoría de los proyectos se sitúan en un estado inicial de su evolución. Es relevante mencionar que muchas de las plataformas que consiguen mayor expansión geográfica forman parte de federaciones de entidades. Es decir, generan una buena interacción con otras organizaciones del cooperativismo de plataforma y de la Economía Social y Solidaria (ESS). Además, tienen un marco de colaboración medio desde la perspectiva del modelo cuádruple hélice. Desde la perspectiva medioambiental, el mayor reto del cooperativismo de plataforma es poder llevar a cabo la visión holística que supone el impacto de una tecnología digital. A pesar de que un buen número de los proyectos promueven productos y servicios para mejorar la eficiencia energética y el consumo responsable, solo una pequeña parte de las plataformas están alojadas en servidores alimentados por energía verde. En este sentido, se ha formulado el ejemplo de Open Food Network, cuya plataforma no está en servidores éticos y verdes por falta tanto de herramientas potentes tanto a nivel de infraestructura como de recursos económicos.

Por último, las plataformas de cooperativismo de plataforma fomentan una gobernanza y un modelo económico democrático, sobre la base de políticas tecnológicas en torno al FLOSS. Sin embargo, los proyectos de cooperativismo de plataforma tienen dos retos principales. Por un lado, fomentar un uso abierto de los datos que enriquezca el conocimiento público en el ámbito del cooperativismo de plataforma. Por otro, la escala de impacto, la capacidad de réplica del proyecto, la sostenibilidad económica, la inclusión e igualdad de género y la cooperación entre agentes.

Financiación: La investigación informada en este documento fue financiada por la Unión Europea, en el marco del programa de investigación e innovación Horizonte 2020, "Platform Labour in Urban Spaces: Fairness, Welfare, Development" (https://project-plus.eu), acuerdo de subvención núm. 822638.

Los puntos de vista y opiniones expresados en esta publicación son responsabilidad exclusiva de los autores y no reflejan necesariamente los puntos de vista de la Comisión Europea / Agencia Ejecutiva de Investigación. 


\section{Bibliografía}

ANWAR, Mohammad \& GRAHAM, Mark (2020): "Between a rock and a hard place: Freedom, flexibility, precarity and vulnerability in the gig economy in Africa", Competition \& Change. DOI: $10.1177 / 1024529420914473$.

BAREA, José (1990): “Concepto y agentes de la economía social”, CIRIEC-España, Revista de Economía Pública, Social y Cooperativa, 8, 109-117.

BAUWENS, Michel (2014, June 16): Book of the day: Solidarity economy alternatives, P2P Foundation. https://blog.p2pfoundation.net/2014/06/16.

BAUWENS, Michel \& KOSTAKIS, Vasilis (2014): "From the communism of capital to capital for the commons: towards an open co-operativism", TripleC: Communication, Capitalism \& Critique, Open Access Journal for a Global Sustainable Information Society, 12(1), 356-361.

DOI: 10.31269/triplec.v12i1.561.

BAUWENS, Michel \& KOSTAKIS, Vasilis (2015): "Towards a new reconfiguration among the state, civil society and the market", Journal of Peer Production, 7. http://peerproduction.net/ issues/issue-7-policies-for-the-commons/peer-reviewed-papers/towards-a-new-reconfiguration-among-the-state-civil-society-and-the-market/

BENKLER, Yochai (2006): The wealth of networks: How social production transforms markets and freedom, Yale University Press.

BIRCHALL, Johnston \& KETILSON, Lou Hammond (2009): Resilience of the Cooperative Business Model in Times of Crisis, Geneva: International Labour Organization.

BIRCHALL, Johnston (2012): "The Comparative Advantages of Member-owned Businesses", Review of Social Economy, 70(3), 263-294.

BOTSMAN, Rachel \& Rogers, Roo (2011): "What's mine is yours: How collaborative consumption is changing the way we live" (Rev. and updated ed), Collins.

BONIN, John; JONES, Derek \& PUTTERMAN, Louis (1993): "Theoretical and Empirical Studies of Producer Cooperatives: Will Ever the Twain Meet?", Journal of Economic Literature, 31(3), 1290-1320.

BURDÍN, Gabriel \& DEAN, Andrés (2009): “New evidence on wages and employment in worker cooperatives compared with capitalist firms", Journal of Comparative Economics, 37(4), 517533. DOI: $10.1016 /$ j.jce.2009.08.001.

CALLE COLLADO, Ángel \& CASADEVENTE, Jose (2015): "Economías sociales y economías para los Bienes Comunes", Otra Economía, 9(16), 44-68. DOI: 10.4013/otra.2015.916.04. 
CASTELLS, Manuel (1996): The rise of the network society, Blackwell Publishers.

CHARLES, Julien; FERRERAS, Isabelle \& LAMINE, Auriane (2020): "A freelancers' cooperative as a case of democratic institutional experimentation for better work: A case study of SMart-Belgium", Transfer: European Review of Labour and Research, 26(2), 157-174.

DOI: $10.1177 / 1024258920919686$.

CHENEY, George; SANTA CRUZ, Iñaki; PEREDO, Ana María \& NAZARENO, Elías (2014): “Worker cooperatives as an organizational alternative: Challenges, achievements and promise in business governance and ownership", Organization, 21(5), 591-603.

CHENG Mingming \& FOLEY, Carmel (2018): "The sharing economy and digital discrimination: The case of Airbnb", International Journal of Hospitality Management, 70, 95-98.

D0I: 10.1016/j.ijhm.2017.11.002.

CODAGNONE, Cristiano; ABADIE, Fabienne; BIAGI, Federico \& Institute for Prospective Technological Studies (2016): The future of work in the 'sharing economy', Publications Office. http://dx.publications.europa.eu/10.2791/431485.

COHEN, Boyd \& KIETZMANN, Jan (2014): "Ride On! Mobility Business Models for the Sharing Economy”, Organization \& Environment, 27(3), 279-296, DOI: 10.1177/1086026614546199.

COHEN, Boyd \& MUÑOZ, Pablo (2016): "Sharing cities and sustainable consumption and production: Towards an integrated framework", Journal of Cleaner Production, 134, 87-97. D0I: 10.1016/j.jclepro.2015.07.133.

COMO, Elena; BATTISTONI, Francesca; SATERIALE, Giulia; PROVVEDI, B; CHOJNICKI, Stefan \& RAPISARDI, Andrea (2015): “Dalla sharing economy all'economia collaborativa: L'impatto e le opportunità per il mondo cooperativo", Quaderni Di Unipolis, 116, http://www.giornalisti. redattoresociale.it/media/287464/unipolis-ricerca-sharing-economy-e-cooperazione.pdf.

COMO, Elena; MATHIS, Agnès; TOGNETTI, Marco \& RAPISARDI, Andrea (2016): "Cooperative Platforms in a European Landscape: An Exploratory Study", 35. https://coopseurope.coop/ sites/default/files/Updated_Paper_Cooperatives\%20Collab\%20Economy.pdf.

DE GROEN, Williem; KILHOFFER, Zachary; LENAERTS, Karolien \& MANDL, Irene (2018): “Employment and working conditions of selected types of platform work, European Foundation for the Improvement of Living and Working Conditions". https://www.eurofound.europa.eu/ publications/report/2018/employment-and-working-conditions-of-selected-types-of-platform-work\#tab-01

DELBONO, Flavio \& REGGIANI, Carlo (2013): "Cooperative firms and the crisis: Evidence from some Italian mixed oligopolies", Annals of Public and Cooperative Economics, 84, 383. 
DE STEFANO, Valerio (2015): "The Rise of the 'Just-in-Time Workforce': On-Demand Work, Crowd Work and Labour Protection in the "Gig-Economy", Comparative Labor Law and Policy Journal. DOI: 10.2139/SSRN.2682602.

DE STEFANO, Valerio (2018): "Negotiating the Algorithm: Automation, Artificial Intelligence and Labour Protection", SSRN Electronic Journal. DOI: 10.2139/ssrn.3178233.

DÍAZ-FONCEA, Millán \& MARCUELLO, Carmen (2014): "The relation between total employment and cooperative employment: A convergence and causality analysis", Spatial Economic Analysis, 9(1), 71.

DÍAZ-FONCEA, Millán \& MARCUELLO, Carmen (2015): "Spatial patterns in new firm formation: are cooperatives different?”, Small Business Economics, 44(1), 171.

DRAHOKOUPIL, Jan \& FABO, Brian (2016): "The Platform Economy and the Disruption of the Employment Relationship", SSRN Electronic Journal. D0I: 10.2139/ssrn.2809517.

DIMMONS (2019): Libro de códigos para el estudio de las cualidades democráticas de las plataformas digitales.

http://dimmons.net/wp-content/uploads/2019/12/PLUS_CODEBOOK_WEB.ods.

EDELMAN, Benjamin \& LUCA, Michael (2014): "Digital Discrimination: The Case of Airbnb. com", Social Science Research Network. DOI: 10.2139/ssrn.2377353.

ESPELT, Ricard (2018): Cooperatives de consum agroecològic de plataforma. El paper de les Tecnologies de la Informació i la Comunicació en el consum cooperatiu de productes agroecològics. http://hdl.handle.net/10803/664225.

EUM, Hyung-sik (2017): Cooperatives and employment: second global report, CICOPA.

FORAMITTI, Joël; VARVAROUSIS, Angelos \& KALLIS, Giorgos (2020): "Transition within a transition: How cooperative platforms want to change the sharing economy", Sustainability Science, 15(4), 1185-1197. D0I: 10.1007/s11625-020-00804-y.

FUSTER MORELL, Mayo (2016): "A new economy: social, commons, feminist, and environmental", P2P Foundation Blog.

FUSTER MORELL, Mayo (Ed.) (2018): Sharing cities: A worldwide cities overview on platform economy policies with a focus on Barcelona, Editorial UOC. http://www.sharingcitiesaction.net/wp-content/uploads/2018/11/SharingCities_book.pdf

FUSTER MORELL, Mayo (2012): “Horizontes del procomún digital”, Documentación social, 165, 89-102. 
FUSTER MORELL, Mayo \& ESPELT, Ricard (2019): "A Framework to Assess the Sustainability of Platform Economy: The Case of Barcelona Ecosystem", Sustainability, 11(22), 6450.

DOI: $10.3390 /$ su11226450.

FUSTER MORELL, Mayo \& ESPELT, Ricard (2018): “How Much are Digital Platforms Based on Open Collaboration?: An analysis of technological and knowledge practices and their implications for the platform governance of a sample of 100 cases of collaborative digital platforms in Barcelona", Proceedings of the 14th International Symposium on Open Collaboration - OpenSym '18, 1-5. DOI: 10.1145/3233391.3233970.

FUSTER MORELL, Mayo; ESPELT, Ricard \& RENAU CANO, Melissa (2020): "Sustainable Platform Economy: Connections with the Sustainable Development Goals", Sustainability, 12(18), 7640. DOI: $10.3390 /$ su12187640.

FUSTER MORELL, Mayo; SALCED0, Jorge \& BERLINGUER, Marco (2016): “Debate About the Concept of Value in Commons-Based Peer Production," In BAGNOLI, Franco; SATSIOU, Anna; STAVRAKAKIS, Ioannis; NESI, Paolo; PACINI, Giovanna, WELP, Yanina; TRIOPANIS, Thanassis \& DIFRANZO, Dominic (Eds.), Internet Science, Springer International Publishing, 27-41. DOI: 10.1007/978-3-319-45982-0_3.

GARCIA, Jordi (2002): “Objectiu: Mercat social”, Nexe, 9, 7.

HAMARI, Juho; SJÖKLINT, Mimmi \& UKKONEN, Antti (2016): “The sharing economy: Why people participate in collaborative consumption", Journal of the Association for Information Science and Technology, 67(9), 2047-2059. D0I:10.1002/asi.23552.

Heinrichs, Harald (2013): "Sharing Economy: A Potential New Pathway to Sustainability", GAIA - Ecological Perspectives for Science and Society, 22(4), 228-231. DOI: 10.14512/gaia.22.4.5.

INTERNATIONAL CO-OPERATIVE MONITOR (2015): "International Co-operative Alliance". https://monitor.coop/sites/default/files/WCM_2015\%20WEB.pdf.

IVANOVA, Mirela; BRONOWICKA, Joanna; KOCHER, Eva \& DEGNER, Anne (2018): “The App as a Boss? Control and Autonomy in Application-Based Management", Europa-Universität Viadrina, 27. https://opus4.kobv.de/opus4-euv/frontdoor/index/index/docld/388.

KENNY, Etlyn \& DONNELLY, Rory (2020): "Navigating the gender structure in information technology: How does this affect the experiences and behaviours of women?", Human Relations, 73(3), 326-350.

LAUKKANEN, Minttu \& TURA, Nina (2020): "The potential of sharing economy business models for sustainable value creation", Journal of Cleaner Production, 253.

DOI: $10.1016 /$ j.jclepro.2020.120004. 
LAVILLE, Jean-Louis (2004): Economía Social y Solidaria, Una visión europea, Altamira. https:// www.economiasolidaria.org/biblioteca/economia-social-y-solidaria-una-vision-europea

LECHAT, Noëlle Marie Paule (2007): “Economia social, economia solidária, terceiro setor: Do que se trata?", Civitas - Revista de Ciências Sociais, 2(1), 123. D0I: 10.15448/1984-7289.2002.1.91.

LEGACOOP (2012): "Manifesto: Cooperative Commons".

http://www.cooperativecommons.coop/index.php/en/manifesto

LEHDONVIRTA, Vili (2018): "Flexibility in the gig economy: Managing time on three online piecework platforms", New Technology, Work and Employment, 33(1), 13-29.

D0I: $10.1111 /$ ntwe.12102.

LESSIG, Lawrence (1999): Code and other laws of cyberspace, Basic Books.

MARTIN, Chris (2016): "The sharing economy: A pathway to sustainability or a nightmarish form of neoliberal capitalism?", Ecological Economics, 121, 149-159.

DOI: 10.1016/j.ecolecon.2015.11.027.

MARTINELLI, Francesca; BOZZONI, Samuele; CAROLI, Simone; TAMASCELLI, Francesca \& GUERINI, Giuseppe (2019): "Platform Cooperativism in Italy and in Europe", CIRIEC Working Papers, 19/27, CIRIEC-Université de Liège.

MONZÓN, José Luis (2003): "El cooperativismo en la historia de la literatura económica”, CIRIEC-España, Revista de Economía Pública, Social y Cooperativa, 44, 9-32.

MONZÓN, José Luis (1989): Las cooperativas de trabajo asociado en la literatura económica y en los hechos, Ministerio de Trabajo y Seguridad Social.

MURRAY, Robin (2010): "Co-operation in the Age of Google", Co-operatives UK, 5. https://www.uk.coop/resources/co-operation-age-google.

NACIONES UNIDAS (2015): “Transforming our world: The 2030 Agenda for Sustainable Development". https://sustainabledevelopment.un.org/post2015/transformingourworld.

OSTROM, Elionor (2015): Governing the Commons: The Evolution of Institutions for Collective Action, Cambridge University Press. DOI: 10.1017/CB09781316423936.

PAZAITIS, Alex; KOSTAKIS, Vasilis \& BAUWENS, Michel (2017): "Digital economy and the rise of open cooperativism: The case of the Enspiral Network", Transfer: European Review of Labour and Research, 23(2), 177-192. D0I: 10.1177/1024258916683865.

PETRUZZI, Marina, SHEPPARD, Valerie \& MARQUES, Catarina (2019): “Positioning Airbnb and Fairbnb in the sharing-exchange continuum", Current Issues in Tourism, 1-4.

DOI: $10.1080 / 13683500.2019 .1697650$. 
PEUTER, Greig de \& Dyer-Witheford, Nick (2010): “Commons and Cooperatives", Affinities: A Journal of Radical Theory, Culture, and Action, 4(1), 30-56.

https://ojs.library.queensu.ca/index.php/affinities/article/view/6147

RATNER, Carl (2009): “Cooperativism: A Social, Economic, and Political Alternative to Capitalism”, Capitalism Nature Socialism, 20(2), 44-73. DOI: 10.1080/10455750902941086.

ROELANTS, Bruno; HYUNGSIK, Eum \& TERRASI, Elisa (2014): Cooperatives and employment: a Global Report, CECOP-CICOPA. DOI: 10.1128/CVI.00435-06.

ROSE, Carol (1986): "The Comedy of the Commons: Custom, Commerce, and Inherently Public Property", The University of Chicago Law Review, 53(3), 711. D0I: 10.2307/1599583.

ROSENBLAT, Alex (2018): Uberland: How algorithms are rewriting the rules of work, University of California Press. DOI: 10.1353/llt.2020.0056.

SCHOLZ, Trebor (2014): "Platform Cooperativism vs. The Sharing Economy”, Medium. https://medium.com/@trebors/platform-cooperativism-vs-the-sharing-economy-2ea737f$1 \mathrm{~b} 5 \mathrm{ad}$.

SCHOLZ, Trebor (2016): "Platform cooperativism. Challenging the Corporate Sharing Economy", Rosa Luxemburg Stiftung.

SCHOLZ, Trebor \& SCHNEIDER, Nathan (Eds.) (2016): Ours to hack and to own: The rise of platform cooperativism, a new vision for the future of work and a fairer internet, OR Books.

DOI: $10.2307 / j . c t v 62 h f q 7$

SCHOR, Juliet (2016): "Debating the sharing economy", Journal of Self-Governance and Management Economics, 4(3), 7-22. DOI: 10.22381/JSME4320161.

SCHOR, Juliet \& FITZMAURICE, Connor (2015): "Collaborating and connecting: The emergence of the sharing economy". In REISCH, Lucia \& THØGERSEN, John (Eds.), Handbook of Research on Sustainable Consumption, Edward Elgar Publishing. DOI: 10.4337/9781783471270.

SCHOR, Juliet; FITZMAURICE, Connor; CARFAGNA, Lindsey; ATTWOOD-CHARLES, Will \& POTEAT, Emilie Dubois (2016): "Paradoxes of Openness and Distinction in the Sharing Economy", Poetics, 54, 66-81. DOI: 10.1016/j.poetic.2015.11.001.

SCHWAB DO NASCIMENTO, Fabio, CALLE-COLLADO, Ángel \& MUÑOZ BENITO, Rocío (2020): "Economía social y solidaria y agroecología en cooperativas de agricultura familiar en Brasil como forma de desarrollo de una agricultura sostenible", CIRIEC-España, Revista de Economía Pública, Social y Cooperativa, 98, 189. D0I: 10.7203/CIRIEC-E.98.14161.

SINGER, Paul (1998): Globalização e desemprego: Diagnóstico e alternativas, Contexto. 
SOLEL, Yifat (2019): "If Uber were a Cooperative: A Democratically Biased Analysis of Platform Economy", Law \& Ethics of Human Rights, 13(2), 239-262. DOI: 10.1515/lehr-2019-2007.

SRNICEK, Nick (2017): Platform capitalism, John Wiley \& Sons.

TODOLÍ SIGNES, Adrián (2018): “Judgment designating Deliveroo 'rider' an employee and analysis of its impact on the 'gig economy', Transfer, The European Review of Labour and Research, 24(4), 487-490. D0I: 10.1177/1024258918801516.

URZI BRANCATI, Maria Cesira; PESOLE, Annarosa \& FERNANDEZ MACIAS, Enrique (2020): New evidence on platform workers in Europe: Results from the second COLLEEM survey, European Commission \& Joint Research Centre. DOI: 10.2760/459278.

VANDAELE, Kurt; PIASNA, Agnieszka \& DRAHOKOUPLI, Jan (2019): “Algorithm Breakers' Are not a Different 'Species': Attitudes Towards Trade Unions of Deliveroo Riders in Belgium", SSRN Electronic Journal. DOI: 10.2139/ssrn.3402899.

ZWICK, Austin (2018): "Welcome to the Gig Economy: Neoliberal industrial relations and the case of Uber", GeoJournal, 83(4), 679-691. D0I: 10.1007/s10708-017-9793-8. 